\title{
The status of anxiety state among cancer patients and their relatives during coronavirus disease 2019 (COVID-19) in Hubei, China
}

\author{
Shengli Yang ${ }^{1 \#}$, Huang Yao ${ }^{2 \#}$, Yating Song ${ }^{3}$, Chengrong Shu ${ }^{4}$, Mengni Chen ${ }^{1}$, Guoliang Pi $^{5}$, Min Jin ${ }^{1}$, \\ Renwang Chen ${ }^{1}$, Qiushuang Wang ${ }^{1}$, Jian Chang ${ }^{1}$, Zhigang $\mathrm{Zuo}^{6}$, Jianli Hu ${ }^{1}$ \\ ${ }^{1}$ Cancer Center, Union Hospital, Tongji Medical College, Huazhong University of Science and Technology, Wuhan, China; ${ }^{2}$ Emergency Department \\ of, Liyuan Hospital, Tongji Medical College, Huazhong University of Science and Technology, Wuhan, China; ${ }^{3}$ Hubei Provincial Center for Disease \\ Control and Prevention, Wuhan, China; ${ }^{4}$ Department of Cancer and Nuclear Medicine, School of Clinical Medicine, Hubei University of Science \\ and Technology/Cancer Center, First Affiliated Hospital of Hubei University of Science and Technology, Xianning, China; ${ }^{5}$ Department of Radiation \\ Oncology, Hubei Cancer Hospital, Tongji Medical College, Huazhong University of Science and Technology, Wuhan, China; ${ }^{6}$ Department of \\ Oncology, Renmin Hospital, Hubei University of Medicine, Shiyan, China \\ Contributions: (I) Conception and design: Z Zuo, J Hu, S Yang, G Pi, Y Song; (II) Administrative support: J Hu, Z Zuo, S Yang; (III) Provision \\ of study materials or patients: J Hu, Z Zuo, S Yang, G Pi, M Jin, R Chen; (IV) Collection and assembly of data: C Shu, M Chen, M Jin, R Chen, \\ Q Wang, J Chang; (V) Data analysis and interpretation: Y Song, S Yang, H Yao; (VI) Manuscript writing: All authors; (VII) Final approval of \\ manuscript: All authors. \\ \#These authors contributed equally to this work. \\ Correspondence to: Prof. Jianli Hu. Cancer Center, Union Hospital, Tongji Medical College, Huazhong University of Science and Technology, 1277 \\ JieFang Avenue, Wuhan 430022, China. Email: j15199@126.com; Prof. Zhigang Zuo. Department of Oncology, Renmin Hospital, Hubei University \\ of Medicine, Shiyan, Maojian District, Chaoyangzhong Road 39, Shiyan 442000, China. Email: doctor_zzg@163.com.
}

Background: During the coronavirus disease 2019 (COVID-19) epidemic, tumor patients and their families might suffer from greater psychological stress as a result of anxiety or other psychological disorders. We conducted an online study during the epidemic to explore the mental state of tumor patients and their families during this extraordinary time.

Methods: A cross-sectional survey was carried out. Questionnaires were distributed through the WeChat "Questionnaire Star" network. The snowball sampling technique was adopted and further promoted by subjects who had completed the questionnaire.

Results: A total of 1,030 valid questionnaires were collected. There were $609(59.13 \%)$ tumor patients and $421(40.87 \%)$ family members. One hundred and fifty-six (15.15\%) subjects had anxiety, among which $65(6.31 \%)$ had moderate to severe anxiety. Single-factor analysis indicated that age ( $>60$ years old), the farmer occupation, and a high sleep disorder assessment score were risk factors for anxiety, while the latter two might also be independent risk factors, as suggested by multi-factor analysis. Infrequent contact with doctors was an independent risk factor for cancer patients, while uninterrupted anti-tumor therapy was an independent protective factor. $40.19 \%$ of the subjects expressed a need for psychosocial support during the COVID-19 period.

Conclusions: The COVID-19 outbreak resulted in tumor patients and their relatives experiencing greater psychological pressure than usual, and patients were more worried about anti-tumor treatment and disease progression impacted by the epidemic. Both groups had a significant need for psychosocial help.

Keywordsı Coronavirus disease 2019 (COVID-19); cancer patients; families; anxiety; confounding factor

Submitted Feb 03, 2021. Accepted for publication Apr 21, 2021.

doi: $10.21037 /$ apm-21-745

View this article at: http://dx.doi.org/10.21037/apm-21-745

^ ORCID: 0000-0002-2068-7618. 


\section{Introduction}

In December 2019, a novel coronavirus disease (COVID-19) outbreak occurred $(1,2)$. This pathogen is the seventh newly discovered coronavirus that can infect humans. It was officially named "2019 novel coronavirus (2019-nCoV)" by the World Health Organization on January 12, 2020 (3), yet the origin of its biological transmission has not been definitely clarified. Over the past few months, the domestic epidemic in China has essentially been controlled through effective prevention and control measures. Unfortunately, new outbreaks are gradually occurring in many other countries around the world, and COVID-19 has become a pandemic, and its worldwide impact is not limited to the threat that it poses to human health.

$2019-\mathrm{nCoV}$ is generally susceptible to the population, and most have a good prognosis, except for the elderly and those with chronic underlying diseases (4). During the COVID-19 epidemic, Wang et al. conducted a large-scale psychological survey in China at the end of January 2020 , and found that $53.8 \%$ the respondents considered that $2019-\mathrm{nCoV}$ had caused moderate to severe psychological problems, partly manifested as anxiety and even depression (5).

In order to effectively prevent and control the COVID-19 outbreak, China has imposed city closures, especially in Wuhan city, and strictly controlled the mobility of the population. These measures were bound to have an impact on the regular treatment of tumor patients and even cross-regional medical treatment. Due to the invasion and consumption of cancer foci, tumor patients are likely to have low immunity and multi-organ damage, making them a high-risk susceptible population for 2019-nCoV with a worse prognosis. Studies have found that for tumor patients, the risk of psychological disorders, such as depression and anxiety, was several times higher than that of the general population $(6,7)$. Moreover, psychological disorders could aggravate tumor progression (8-10). Therefore, during the COVID-19 epidemic, tumor patients, as a group of special subjects with a higher incidence of psychological problems, were easily affected when regular treatment could not be carried out on schedule. Furthermore, being relatively susceptible to 2019-nCoV, these patients might be more likely to suffer from psychological stress, anxiety, and other psychological problems. At the same time, the increased care and other difficulties faced by the family members of cancer patients might also present a risk of anxiety. To our knowledge, there are few related studies on this topic.
In order to investigate the anxiety status of tumor patients and their relatives during the COVID-19 epidemic, and to explore the corresponding intervention strategies, we conducted a large psychological survey in the form of a questionnaire. We present the following article in accordance with the SURGE reporting checklist (available at http://dx.doi.org/10.21037/apm-21-745).

\section{Methods}

\section{Survey objects}

The majority of cancer patients and their families who participated in this study were in Hubei province of China. The study was conducted in accordance with the Declaration of Helsinki (as revised in 2013). The study was approved by the Ethics Committee of Tongji Medical College, Huazhong University of Science and Technology (No.: IORG0003571) and informed consent was taken from all individual participants.

\section{Survey methods}

A cross-sectional survey was performed using a self-made questionnaire. The doctors at the Cancer Center, Union Hospital, Tongji Medical College, Huazhong University of Science and Technology, distributed the questionnaire online via the WeChat "Questionnaire Star", patients and their caregivers respond anonymously and free of charge. The snowball sampling technique was adopted and further promoted by the subjects who had completed the questionnaire. From March 4, 2020, to March 20, 2020, 1,030 questionnaires were collected in total, and all were sufficiently filled in, with an effective questionnaire return ratio of $100 \%$.

\section{Questionnaire design}

The questionnaire was divided into five sections, including general demographic characteristics (sex, age, marital status, city, education, occupation, whether infected with COVID-19, etc.; a total of 10 items); tumorrelated information (occurrence time of tumor, current treatment stage, whether treatment was interrupted due to COVID-19, etc.; a total of five items); sleep quality during the last 2 weeks (via a self-rating scale scoring system); anxiety state [using the Self-rating Anxiety Scale (SAS)]; and a survey of subjective feelings. 


\section{Sleep and anxiety assessment methods}

Sleep disorder assessment was conducted using a scoring system, with five options for each question corresponding to the score (no $=0$, mild $=1$, moderate $=2$, severe $=3$, and extreme $=4)$. Finally, the total score was calculated for further assessment.

SAS was used for anxiety assessment. The options corresponded to the score (no or little $=1$, a small part of the time $=2$, most of the time $=3$, almost the whole day $=4$ ), and the total score was calculated. score more than over 50 points indicated anxiety, 50-59 points denoted mild anxiety, 60-69 points represented moderate anxiety, and over 70 points indicated severe anxiety.

\section{Statistical methods}

Counting data were described as a frequency and percentage (\%). The measurement data did not conform to a normal distribution and was described as a median. The characteristics of each group were compared using Wilcoxon's rank sum test for sleep test score and the chi-square test for other variables. In addition, logistic regression was used for univariate and multivariate analysis, represented as OR (95\% confidence interval CI). Multiple imputation was used to impute missing values. $\mathrm{P}$ value less than 0.05 was considered statistically significant.

\section{Results}

\section{Relations among general demographic characteristics, tumor-related conditions, sleep, and anxiety}

A total of 1,030 valid questionnaires were collected (the effective return rate was 100\%), among which 609 (59.13\%) were tumor patients and $421(40.87 \%)$ were their family members. There were $156(15.15 \%)$ subjects with anxiety, including $91(8.83 \%)$ with mild anxiety, 40 (3.88\%) with moderate anxiety, and $25(2.43 \%)$ with severe anxiety. Anxiety was observed in 86 tumor patients and in 70 family members.

Among all subjects, there were 457 (44.37\%) males and $573(55.63 \%)$ females. Six $(0.58 \%)$ subjects were less than 18 years old, $838(81.36 \%)$ were $18-60$ years old, and 186 $(18.06 \%)$ were over 60 years of age. $873(84.76 \%)$ were married, and 157 (15.24\%) were unmarried, divorced, or widowed. There were $693(67.28 \%)$ subjects living in Wuhan, 273 (26.50\%) living in other areas of Hubei province besides Wuhan, and $64(6.21 \%)$ living outside
Hubei province. 456 (44.27\%) subjects had a bachelor degree or above, while 574 (55.73\%) had a lower degree. There were $71(6.89 \%)$ medical staff and 959 (93.11\%) other professionals. $8(0.78 \%)$ cancer patients and $7(0.68 \%)$ family members were infected with COVID-19. Singlefactor analysis indicated that sex $(\mathrm{P}=0.0393)$, age $(\mathrm{P}=0.0320)$, place of residence $(\mathrm{P}=0.0254)$, educational background $(\mathrm{P}<0.0001)$, occupation $(\mathrm{P}=0.0034)$, and sleep disorder assessment $(\mathrm{P}<0.0001)$ were statistically significant factors. Among these, $>60$ years old, the farmer occupation, and higher sleep disorder assessment score had an odds ratio $(\mathrm{OR})>1$, indicating that these were risk factors for anxiety. However, factors including sex (females), residence in other areas of Hubei province besides Wuhan, middle school or technical secondary school degree, bachelor degree or above had an $\mathrm{OR}<1$, indicating that these were protective factors (Table 1).

Among the 609 tumor patients, 91 (14.94\%) had a tumor duration of 3 months, 138 (22.66\%) had a tumor duration of 6 months, and $380(62.40 \%)$ had a tumor duration of 1 year or more. There were $14(2.30 \%)$ patients undergoing earlystage treatment, $346(56.81 \%)$ patients in the treatment period, $51(8.37 \%)$ patients in the late recovery period, and $198(32.51 \%)$ patients in the regular follow-up stage. 288 $(47.29 \%)$ patients experienced treatment interruption due to COVID-19, while 321 (52.71\%) did not. There were $477(78.33 \%)$ patients that were in frequent contact with doctors and $132(21.67 \%)$ patients that were not. Singlefactor analysis suggested that factors including treatment interruption $(\mathrm{P}=0.0008)$, regular contact with doctors $(\mathrm{P}=0.0005)$, and treatment methods $(\mathrm{P}=0.0306)$ were statistically significant. Among these, infrequent contact with doctors had an OR $>1$, indicating that this was a risk factor for anxiety. However, treatment without interruption had an $\mathrm{OR}<1$, signifying that this was a protective factor (Table 2).

\section{Multi-factor analysis}

As shown in Table 2, after adjustment for covariates on multivariate analysis, education background $(\mathrm{P}=0.007)$, occupation $(\mathrm{P}=0.015)$, and sleep disorder assessment score $(\mathrm{P}<0.001)$ were identified as independent predictors for anxiety in tumor patients and their relatives. Of these, the farmer occupation and a high sleep disorder assessment score had an $\mathrm{OR}>1$, indicating that they were independent risk factors for anxiety, while the OR $<1$ of middle school or technical secondary school degree indicated that this was an 


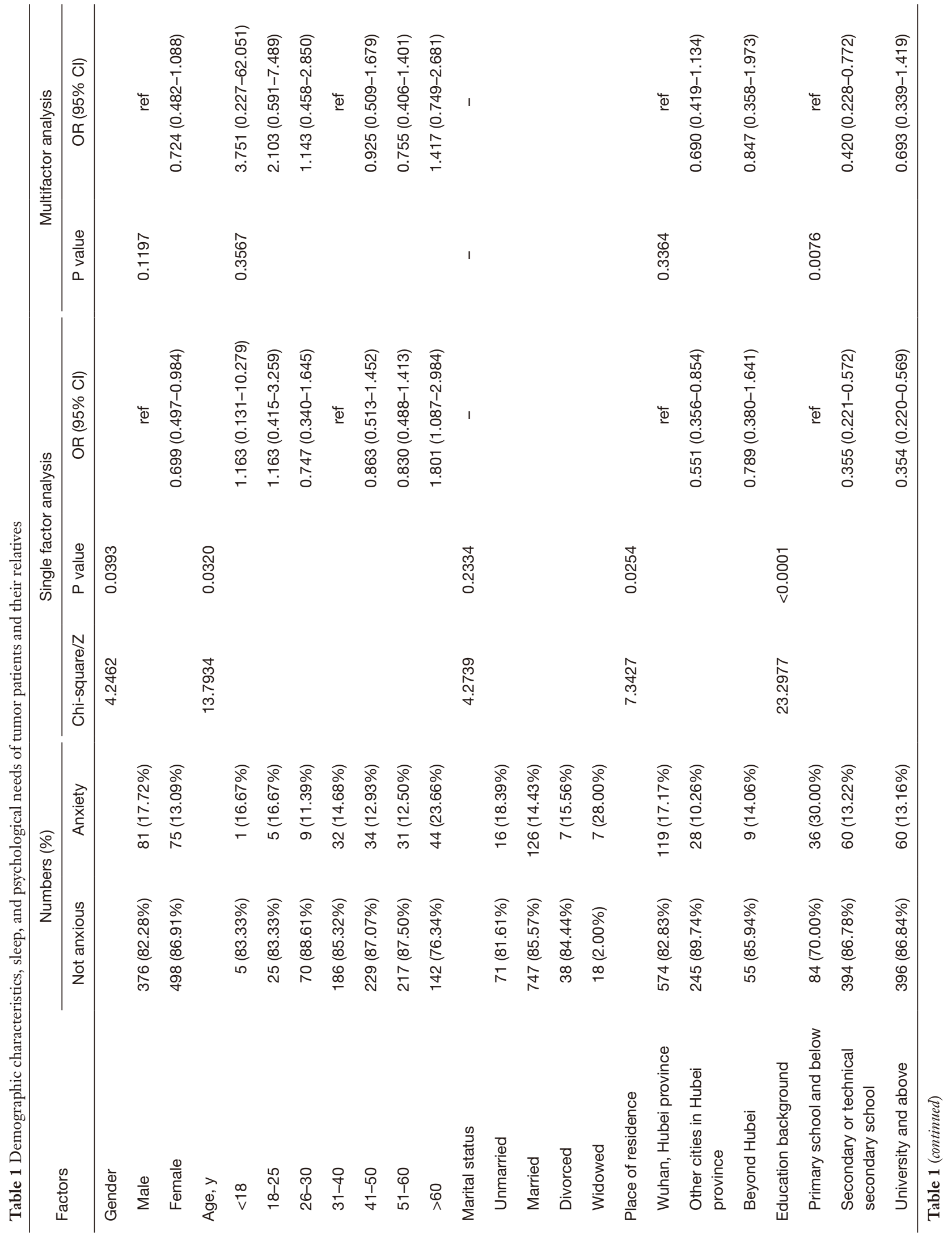




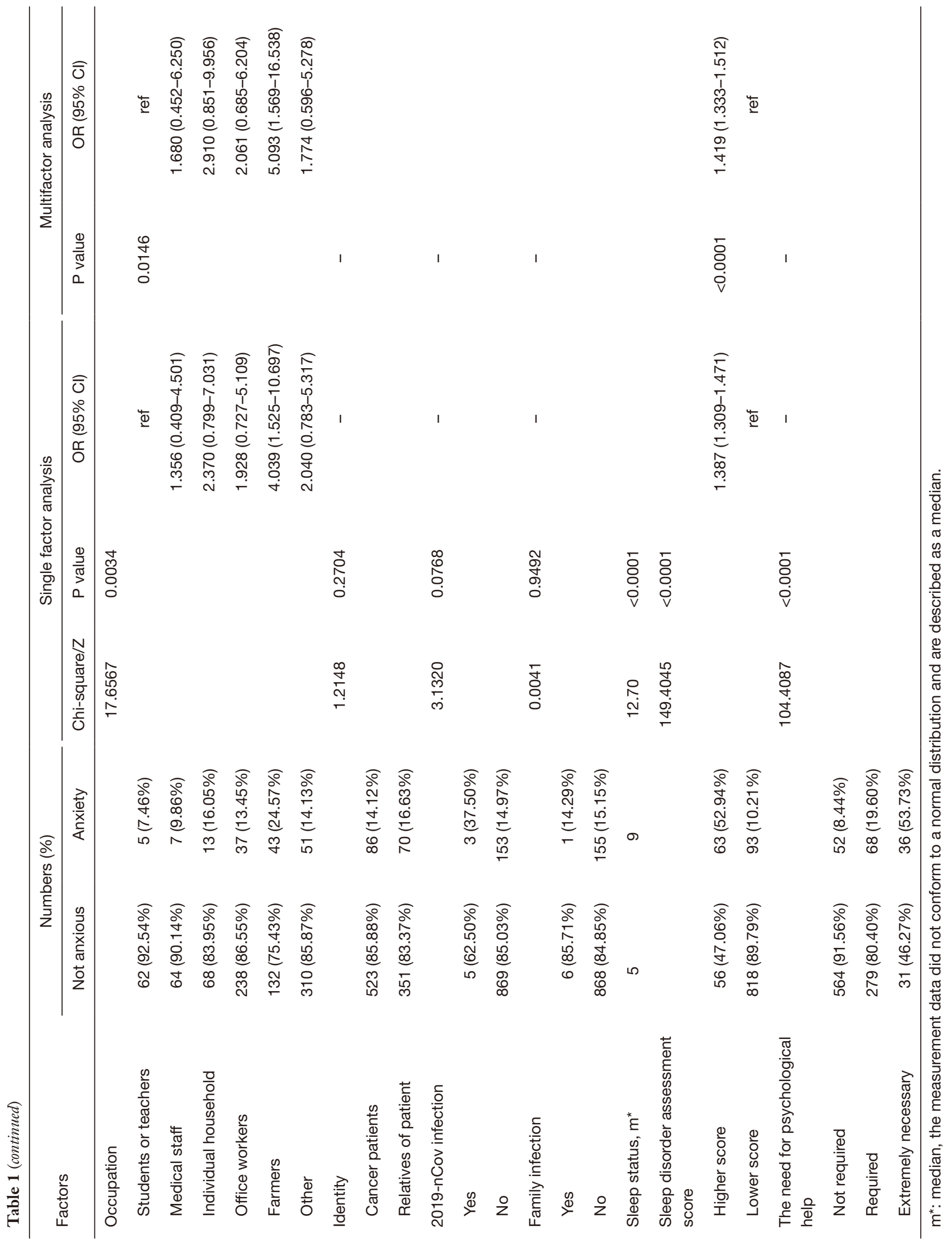




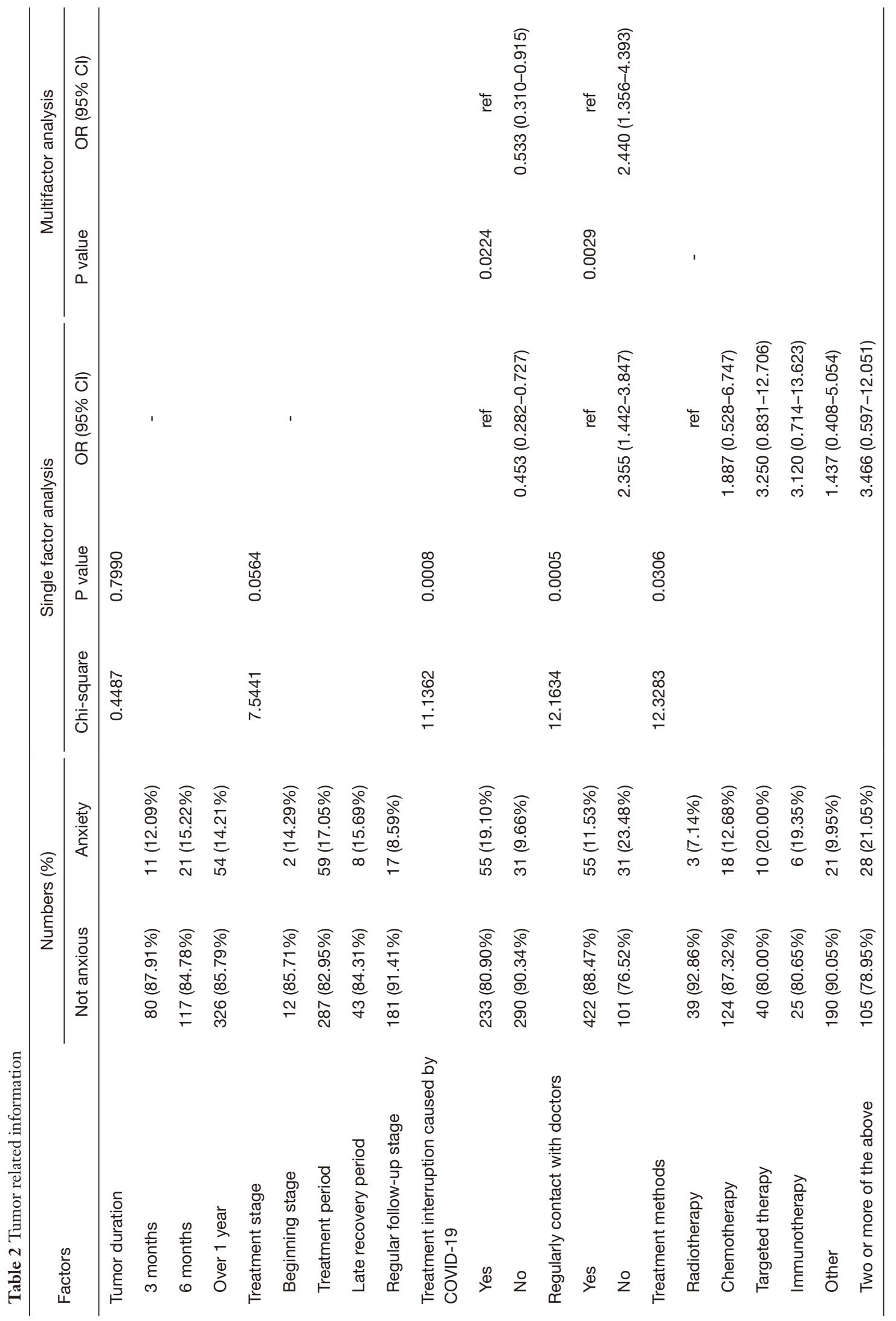


independent protective factor (Table 1). For tumor patients, treatment interruption and regular contact with doctors were statistically significant factors. Of these, infrequent contact with doctors had an $\mathrm{OR}>1$, indicating that this was an independent risk factor for anxiety, while treatment without interruption had an $\mathrm{OR}<1$, signifying that it was an independent protective factor (Table 2).

\section{Subjective feelings and suggestions}

Prior to the outbreak of COVID-19, 36.95\% of the patients reported moderate or greater psychological distress caused by the tumor (moderate $22.99 \%$, severe $8.70 \%$, and extreme $5.25 \%$ ). Meanwhile, in the period of COVID-19, this proportion rose to $43.84 \%$ (moderate $24.79 \%$, severe $10.84 \%$, extreme $8.21 \%$ ). $66.50 \%$ of the subjects reported that they felt the trend of aggravation and suffered different degrees of fear (mild $31.53 \%$, moderate $18.56 \%$, severe $9.69 \%$, extreme $6.73 \%)$. Nearly half $(46.96 \%)$ of the patients reported that their access to tumor drugs during the epidemic period was affected. However, in the face of closed hospital administration, most patients (70.94\%) felt less affected $(27.91 \%)$ or not affected $(43.02 \%)$. Singlefactor analysis indicated that the more severe the condition (above) was, the higher the anxiety rate would be (Table 3).

Among all of the subjects, those with psychological help demands accounted for $40.19 \%$, and $6.50 \%$ of them chose "very needed" (Table 1). The types of psychological help that people tended to accept were mainly relaxation and decompression, emotional counseling, and psychological knowledge popularization (Figure 1).

\section{Discussion}

On March 5, 2020, the National Health Commission issued the "Notice on Strengthening the Psychological Assistance and Social Work Services in the Work of Responding to COVID-19" (11), which emphasized that special attention should be given to cancer patients with other primary diseases. Thus, medical staff needed to provide psychological counseling and other services. According to a largescale domestic psychological survey (involving 194 cities) conducted at the end of January (5), more than half of the respondents believed that the epidemic had a moderate to severe psychological impact. Psychological problems have become an issue that should not be ignored in this COVID-19 epidemic.

Psychological problems are quite common among cancer patients. Many previous in-depth studies (12-14) have shown that the factors causing psychological problems in cancer patients include the following: fear of the cancer disease itself; physical discomfort during treatment; lack of normal social life, communication, and interaction; unsatisfied psychological needs; neurotoxicity caused by treatment, which leads to neurocognitive dysfunction; family financial burden, etc. Excessive perception of the disease may also have physiological effects, such as continued pressure leading to activation of the hypothalamic-pituitaryadrenal axis, thereby inducing psychological symptoms and reaching the diagnostic threshold for depression or anxiety. Common physiological side effects of conventional cancer chemotherapy, such as vomiting, hair loss, mucositis and peripheral neuropathy, may also be a source of chronic psychological stress (15). At present, many countries have incorporated psychological assessment into the relevant guidelines and government management regulations for cancer treatment, and have even expanded the scope to caregivers and medical service personnel of cancer patients, with necessary re-evaluations conducted after a certain interval $(16,17)$. In 2016, China also formulated the first edition of the "Guidelines for Oncology Psychotherapy in China" (18).

After investigation, we found that among the 1,030 cancer patients and their families, 156 cases (15.15\%) developed anxiety. Among them, 86 cases were cancer patients, and 70 cases were family members of patients. At present, extensive epidemiological studies tend to believe that women are at higher risk of developing psychological disorders, such as depression (19). However, in the severe acute respiratory syndrome (SARS) epidemic of 2003, Leung GM found that women may be more alert and would take preventive measures earlier. Preventive measures (such as hand hygiene, wearing masks, etc.) have been shown to have a positive effect on psychological state during the COVID-19 epidemic (20). In this psychological survey, we also found that women were less likely to have anxiety than men, which reflects that the general situation during the COVID-19 epidemic was more specific than usual. Also, in the area with the highest epidemic density, the proportion of fear and anxiety in the population was higher than that of other surrounding areas. In this study, the sample size of the population outside Hubei Province was small, and no statistical significance was observed, which was also a limitation of this study. According to the latest research, education level, age, and other factors are also closely related to the occurrence of anxiety (7). This study 
Table 3 Subjective investigation on tumor patients

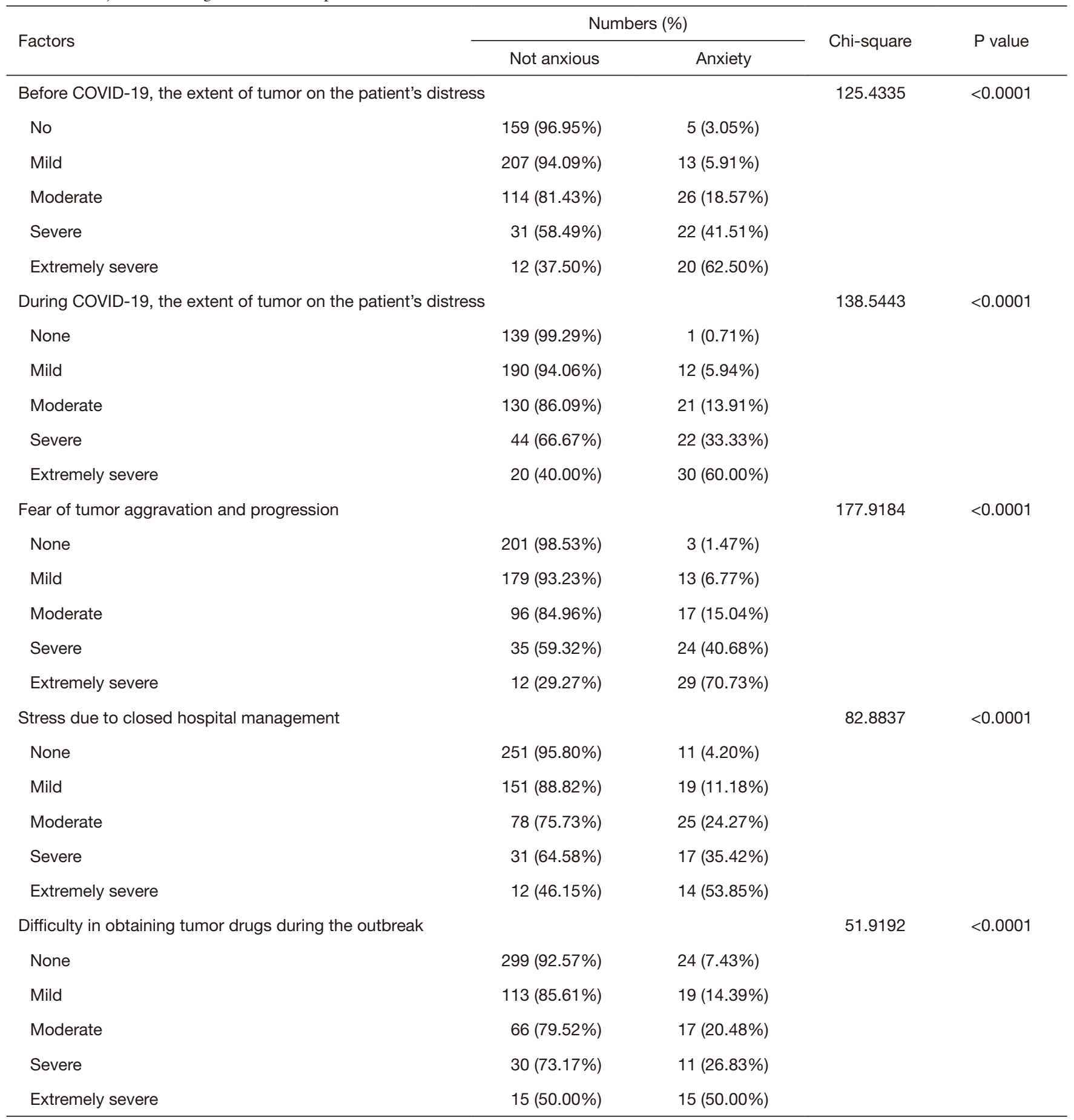

found that cancer patients or their family members who were over 60 years of age or working as farmers were more likely to have anxiety, while those who were educated in middle school or above were less likely to have anxiety. In addition, according to some previous studies and relevant guidelines $(18,21)$, poor sleep quality increased the risk of psychological abnormality, which was consistent with the results of this study. The multi-factor analysis in this study 
700

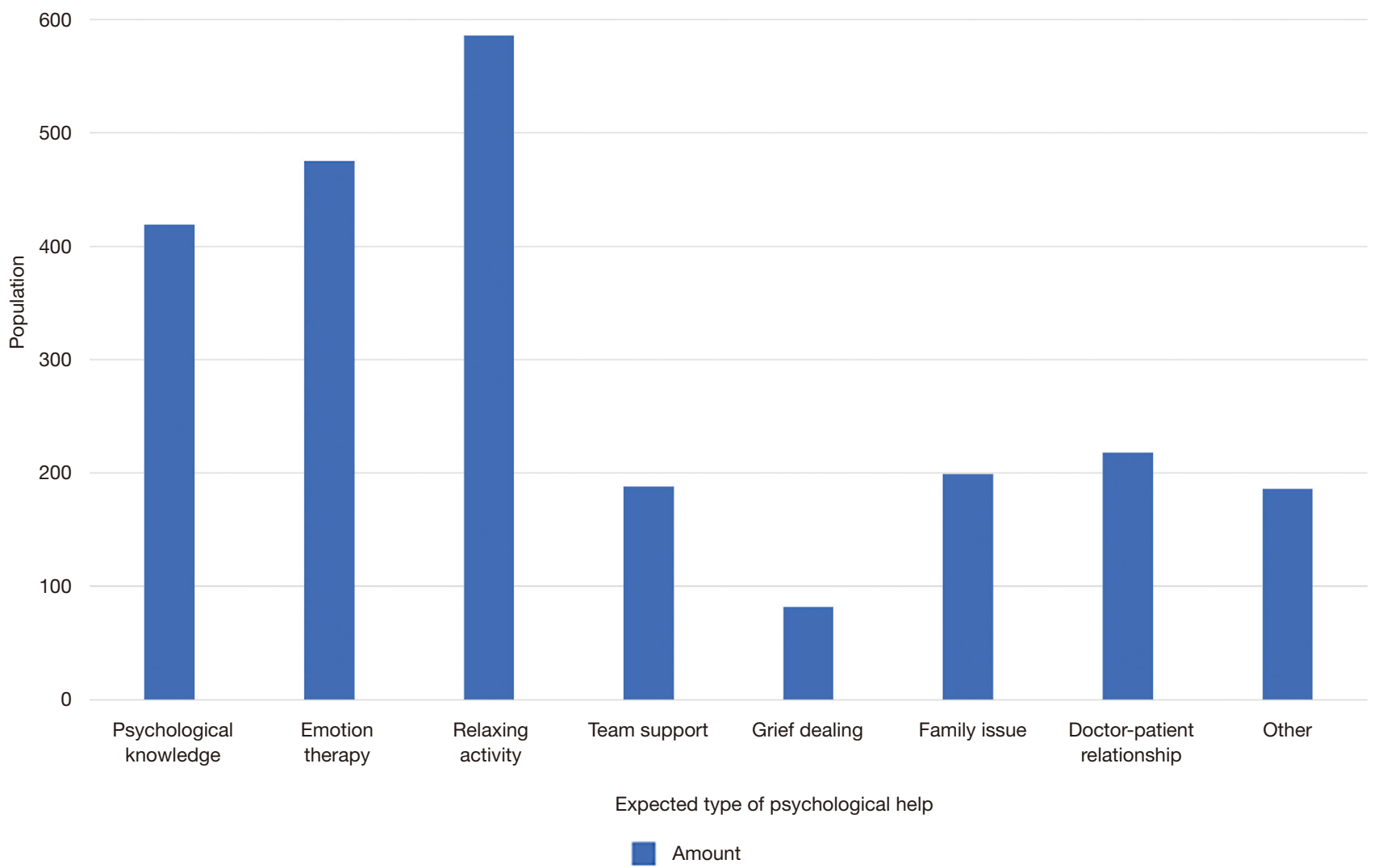

Figure 1 Types of psychological help that tumor patients and their families wished to receive.

also suggested that for cancer patients and their families, the farmer occupation and higher sleep disorder assessment score were independent risk factors for anxiety. Meanwhile, an education level of secondary school (or higher) was an independent protective factor compared to primary school education (or below).

During the disease outbreak, related media reports of deaths and the increasing number of new cases increased anxiety in the community, people's desire for facts escalated, and the lack of clear information further increased fear (22). Research by Wang et al. showed that in the early stage of the COVID-19 epidemic, timely and accurate information on the research progress of the epidemic (especially the number of rehabilitation cases or the latest information on drug and vaccine research) contributed to relieving people's anxiety and reducing psychological pressure (5). Our study began on March 4th, at which time the domestic epidemic situation had been basically effectively controlled, and the epidemic was entering into the middle or later stages. Progress has since been made in the study of virus characteristics and effective drugs/vaccines. Case data related to the epidemic has also been significantly improved and become open and transparent. The understanding of the population regarding the current epidemic situation and the disease has been strengthened. The subjective perception survey in this study found that the proportion of people who attributed their anxiety to the lack of sufficient knowledge about new coronary pneumonia was only $17.57 \%$. In addition, we found that the overall rate of anxiety was $15.15 \%$, which was less than that in the earlier studies (5). At the same time, we found that people over the age of 60 and those who worked as farmers were more prone to develop anxiety in epidemic situations, while those with high school education (or above) were relatively 
less likely to have anxiety. The first two groups of people had relatively poor access to and acceptance of the latest epidemic-related knowledge or information, while the latter group could be described as activists in this information age. This also provided strong evidence that research progress, information transparency, and the improvement of the epidemic situation contributed to relieving public anxiety.

In order to effectively prevent and control the spread of the COVID-19 epidemic, our country adopted comprehensive blockade and isolation measures, and thus, regular anti-tumor treatment and follow-up would inevitably be affected. Our multi-factor analysis demonstrated that under the influence of the epidemic, lack of frequent contact with doctors was an independent risk factor for anxiety, while the uninterrupted treatment of cancer was an independent protective factor. The subjective feelings survey showed that following the outbreak of the epidemic, the proportion of psychological distress caused by tumors increased from $36.95 \%$ to $43.84 \%, 66.50 \%$ of the subjects felt that the tumor disease was aggravated and they were afraid of it, and nearly half (46.96\%) of the patients were affected in obtaining anti-tumor drugs. As the degree of worry and fear of the aforementioned situation increased, the proportion of anxiety also increased correspondingly, and the difference was statistically significant. Irrespective of cancer patients or their families, $40.19 \%$ of participants felt that they needed psychosocial help during the epidemic, of which $6.50 \%$ thought it was extremely necessary, reminding us that further social and psychological support work is critical at present. According to the subjective survey in this study, it may be necessary to start from the aspects of relaxation and decompression, emotional counseling, and popularization of psychological knowledge. For patients with novel coronavirus pneumonia, we have special psychologists to assess their psychological state, then give psychological counseling and psychological intervention.

\section{Conclusions}

In the current COVID-19 epidemic, it is necessary to pay attention to the psychological needs of cancer patients, and extend the scope of care to their families. In order to establish a comprehensive and scientific system to promptly identify and focus on high-risk groups, the key factors to be considered include the following: gender, age, occupation, location, and sleep condition. Of these, sleep disorder and occupation as a farmer may be the independent risk factors for anxiety, and higher education could be the independent protective factor. Meanwhile, we also need to pay more attention to whether the epidemic situation affected antitumor treatment of patients. Infrequent contact with doctors was an independent risk factor for anxiety, while uninterrupted tumor treatment was an independent protective factor. In the middle and later stages of the epidemic, the main source of anxiety for cancer patients may be the negative impact of the COVID-19 epidemic on their normal cancer treatment; the degree of worry was greater than the fear of the epidemic itself, and patients and their families had a significant need for social and psychological help. Research on the mental health of cancer patients has a long way to go. The COVID-19 epidemic was an important point that may trigger a sudden change in the mental state of cancer patients.

\section{Acknowledgments}

Funding: This work was supported by the Cancer Psychology Committee of Hubei Cancer Association and undergraduate innovation and entrepreneurship training program of China (202010487059) and of Hubei province (S202010487036).

\section{Footnote}

Reporting Checklist: The authors have completed the SURGE reporting checklist. Available at http://dx.doi. org/10.21037/apm-21-745

Data Sharing Statement: Available at http://dx.doi. org/10.21037/apm-21-745

Conflicts of Interest: All authors have completed the ICMJE uniform disclosure form (available at http://dx.doi. org/10.21037/apm-21-745). The authors have no conflicts of interest to declare.

Ethical Statement: The authors are accountable for all aspects of the work in ensuring that questions related to the accuracy or integrity of any part of the work are appropriately investigated and resolved. The study was conducted in accordance with the Declaration of Helsinki (as revised in 2013). The study was approved by the Ethics Committee of Tongji Medical College, Huazhong University of Science and Technology (No.: IORG0003571) and informed consent was taken from all individual participants. 
Open Access Statement: This is an Open Access article distributed in accordance with the Creative Commons Attribution-NonCommercial-NoDerivs 4.0 International License (CC BY-NC-ND 4.0), which permits the noncommercial replication and distribution of the article with the strict proviso that no changes or edits are made and the original work is properly cited (including links to both the formal publication through the relevant DOI and the license). See: https://creativecommons.org/licenses/by-nc-nd/4.0/.

\section{References}

1. Zhu N, Zhang D, Wang W, et al. A Novel Coronavirus from Patients with Pneumonia in China, 2019. N Engl J Med 2020;382:727-33.

2. Cheng VCC, Wong S-C, To KKW, et al. Preparedness and proactive infection control measures against the emerging novel coronavirus in China. J Hosp Infect 2020;104:254-5.

3. National Health Commission of the People's Republic of China. Notice of the National Health Commission on the revision of the English naming of COVID - 19. 2020.

4. National Health Commission of the People's Republic of China. Notice on the issuance of COVID-19 protocol (trial seventh edition). 2020.

5. Wang C, Pan R, Wan X, et al. Immediate Psychological Responses and Associated Factors during the Initial Stage of the 2019 Coronavirus Disease (COVID-19) Epidemic among the General Population in China. Int J Environ Res Public Health 2020;17:1729.

6. Pitman A, Suleman S, Hyde N, et al. Depression and anxiety in patients with cancer. BMJ 2018;361:k1415.

7. Niedzwiedz CL, Knifton L, Robb KA, et al. Depression and anxiety among people living with and beyond cancer: a growing clinical and research priority. BMC Cancer 2019;19:943.

8. Zhu J, Fang F, Sjölander A, et al. First-onset mental disorders after cancer diagnosis and cancer-specific mortality: a nationwide cohort study. Ann Oncol 2017;28:1964-9.

9. Massetti GM, Thomas CC, King J, et al. Mental Health Problems and Cancer Risk Factors Among Young Adults. Am J Prev Med 2017;53:S30-9.

10. Klaassen Z, Wallis CJD, Goldberg H, et al. The impact of psychiatric utilisation prior to cancer diagnosis on survival of solid organ malignancies. Br J Cancer 2019;120:840-7.

11. National Health Commission of the People's Republic of
China. Notice on strengthening psychological assistance and social work services in response to COVID-19 outbreak. 2020

12. Wen S, Xiao H, Yang Y. The risk factors for depression in cancer patients undergoing chemotherapy: a systematic review. Support Care Cancer 2019;27:57-67.

13. Ismail MF, Lavelle C, Cassidy EM. Steroid-induced mental disorders in cancer patients: a systematic review. Future Oncol 2017;13:2719-31.

14. Gilligan AM, Alberts DS, Roe DJ, et al. Death or Debt? National Estimates of Financial Toxicity in Persons with Newly-Diagnosed Cancer. Am J Med 2018;131:1187-99.e5.

15. SMITH HR. Depression in cancer patients: Pathogenesis, implications and treatment (Review). Oncol Lett 2015;9:1509-14.

16. American Cancer Society \& National Comprehensive Cancer Network. Distress Treatment guidelines for patients Version II. 2016.

17. American College of Surgeons' Commission of Cancer. Cancer Program Standards: Ensuring Patients-Centered Care. 2015.

18. Tang L. Interpretation of the Chinese Psychosocial Oncology Therapy Guidelines for Cancer Patients. Medicine \& Philosophy 2016;11:25-7.

19. Lim GY, Tam WW, Lu Y, et al. Prevalence of Depression in the Community from 30 Countries between 1994 and 2014. Sci Rep 2018;8:2861.

20. Leung GM. The impact of community psychological responses on outbreak control for severe acute respiratory syndrome in Hong Kong. J Epidemiol Community Health 2003;57:857-63.

21. Jia Y, Liu L, Sheng C, et al. Increased Serum Levels of Cortisol and Inflammatory Cytokines in People With Depression. J Nerv Ment Dis 2019;207:271-6.

22. Rubin GJ, Wessely S. The psychological effects of quarantining a city. BMJ 2020;368:m313.

(English Language Editor: A. Kassem)

Cite this article as: Yang S, Yao H, Song Y, Shu C, Chen M, Pi G, Jin M, Chen R, Wang Q, Chang J, Zuo Z, Hu J. The status of anxiety state among cancer patients and their relatives during coronavirus disease 2019 (COVID-19) in Hubei, China. Ann Palliat Med 2021;10(4):4601-4611. doi: 10.21037/apm-21745 